\title{
DiGeorge syndrome: part of CATCH 22
}

\author{
D I Wilson, J Burn, P Scambler, J Goodship
}

\begin{abstract}
DiGeorge syndrome (DGS) comprises thymic hypoplasia, hypocalcaemia, outflow tract defects of the heart, and dysmorphic facies. It results in almost all cases from a deletion within chromosome $22 q 11$. We report the clinical findings in 44 cases. We propose that DiGeorge syndrome should be seen as the severe end of the clinical spectrum embraced by the acronym CATCH 22 syndrome; Cardiac defects, Abnormal facies, Thymic hypoplasia, Cleft palate, and $H y$ pocalcaemia resulting from $22 q 11$ deletions.
\end{abstract}

(f Med Genet 1993;30:852-6)

In 1965 DiGeorge described a child with hypoparathyroidism and recurrent infections along with three necropsy cases of absent thymus and parathyroid glands at an immunology meeting. ${ }^{1}$ The phenotype of the syndrome that was named after him was widened to include congenital heart disease and facial dysmorphism. The first clue to the aetiology of DGS came when de la Chapelle reported a family with a chromosome translocation, $t(20 ; 22)(q 11 ; q 11)$, in which four subjects with unbalanced products had DGS. ${ }^{2}$ It is now well established that the vast majority of cases result from deletions within chromosome $22 \mathrm{q} 11 .^{3-5}$ Here we report the phenotypic features in our series of 44 cases of DGS and set this in the broader clinical context of 22q11 deletions, for which we now propose the acronym CATCH 22 syndrome.

\section{Subjects}

This clinical study is based on 44 children with DiGeorge syndrome (21 female and 23 male) who were referred by members of the British Paediatric Association, the British Paediatric Cardiac Association, and the Clinical Genetics Society. Children were considered to have fulfilled the diagnostic criteria if they had at least three out of the following four features: cardiac defect, hypocalcaemia, evidence of thymus hypoplasia, and dysmorphic features. One of these children has features of both Noonan syndrome and DiGeorge syndrome and has already been described in detail. ${ }^{6}$

\section{Cardiac abnormalities}

Each child was investigated in a regional paediatric cardiothoracic centre by echocardio- graphy or cardiac catheterisation or both. Of the 44 children, 42 had a cardiac defect (table 1). In 35 cases the cardiac anatomy was confirmed at operation or necropsy. The majority had obstructive lesions, predominantly affecting the left outflow tract. Eighteen had interrupted aortic arch (IAA) type $B$ and one IAA type C. Two had a hypoplastic left heart and one coarctation of the aorta.

The right outflow tract was also affected in a significant proportion of children, the lesions again being primarily obstructive. Five had pulmonary atresia with a ventricular septal defect (VSD), three had tetralogy of Fallot, one had double outlet right ventricle, and two had mild isolated pulmonary valve stenosis.

It was common for the children to have more than one structural defect, for example, VSD in association with IAA. There were three children who had isolated septal defects. These were a VSD, an atrioventricular septal defect, and a secundum atrial septal defect.

A significant proportion of children were found to have an aberrant right subclavian artery or a right aortic arch. For example, GOS4 presented with hypocalcaemia but subsequently developed dysphagia secondary to

Table 1 Cardiac abnormalities. The major defects are listed in bold print and the associated abnormalities in the indented lists.

\begin{tabular}{l}
\hline IAA(type B)/VSD \\
IAA(type C)/VSD \\
RAA \\
ARSA \\
Bicuspid AoV \\
ASD \\
Muscular VSD \\
TV/AoV hypoplasia \\
Truncus, AVSD \\
Truncus \\
RAA \\
ARSA \\
Small LPA \\
Pulmonary atresia/VSD \\
RAA \\
ALSA \\
MAPCA \\
Vascular ring \\
Fallot's tetralogy \\
ARSA \\
RAA \\
DORV \\
Absent PV \\
Lhypoplastic heart \\
Mild PVS \\
ASD (secundum) \\
AVSD/Coarctation \\
VSD (isolated) \\
ARSA \\
Coarctation
\end{tabular}

$\mathrm{DORV}=$ double outlet right ventricle; $\mathrm{ARSA}=$ aberrant right subclavian artery; ALSA = aberrant left subclavian artery; subclavian artery; ALSA = aberrant left subclavian artery; PVS $=$ pulmonary valve stenosis; $A o V=$ aortic valve; $T V=$ tricuspid valve; RAA = right aortic arch; $\mathrm{LPA}=$ left pulmonary artery; $\mathrm{MAPCA}=$ multiple anomalous pulmonary collateral arteries. 
an aberrant right subclavian artery. Although these abnormalities are clinically less severe than major obstructive lesions, they still represent abnormal development of the pharyngeal arch arteries. IAA type B may result from maldevelopment of the fourth left branchial arch artery and an aberrant right subclavian artery may arise from maldevelopment of the fourth right branchial arch artery. These two lesions are likely to have a similar embryological origin but have very different clinical manifestations.

\section{Hypocalcaemia}

Children were considered to be hypocalcaemic if the total ionised serum calcium was less than the normal range for the laboratory performing the analysis. Forty children had documented hypocalcaemia out of the 44 cases of DGS. The serum calcium was not measured in one child as it was clear that she would die rapidly from her cardiac lesion. In three children hypocalcaemia was not documented despite two having received intensive monitoring.

In four children the presenting feature was a seizure secondary to hypocalcaemia. A further nine had a seizure after the diagnosis of a cardiac defect.

Information about the onset of hypocalcaemia was available in 24 cases. In 20 of these, onset was within the first 14 days of life but in one child it was first documented at 5 years of age when a child presented with hypocalcaemic convulsions six weeks after operative repair of Fallot's tetralogy.

The hypocalcaemia resolved in 26 of the 40 cases. Four of the hypocalcaemic children died. In the remaining 10 cases treatment has continued. Parathormone was measured in nine children when they were hypocalcaemic and found to be inappropriately low in eight of these.

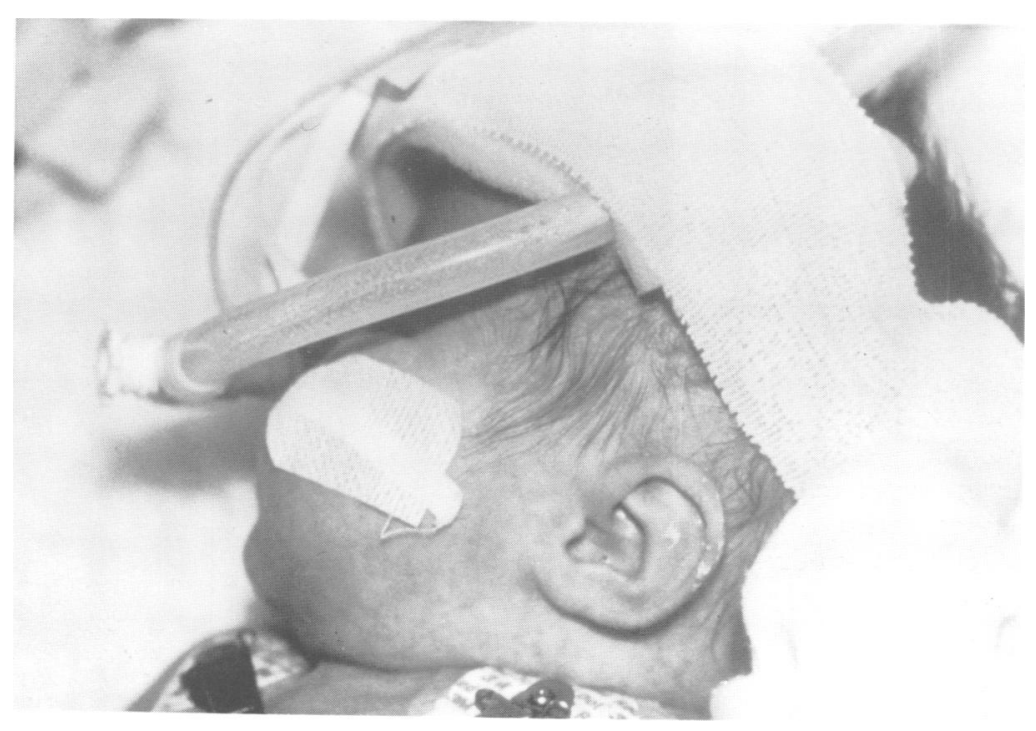

Figure 1 This figure shows the difficulty in assessing the dysmorphic features in the sick neonate, though the appearance of the ears is helpful in this ventilated neonate.

\section{Thymic abnormalities}

Thirty-three children had either low $\mathrm{T}$ cells or thymus hypoplasia. Five children were considered to have normal thymus/immune function and in six children the data were inadequate to assess immune function. The thymus gland could not be identified in 18 children undergoing sternotomy for repair of a cardiac defect or at necropsy and in six cases the thymus was considered inappropriately small even accounting for the shrinkage that occurs in severely ill neonates. Interestingly there were several children in whom no thymus was identified at operation whose $T$ cell numbers were only just below the lower limit of normal. The assessment of the thymus gland by chest $x$ ray was often found to be incorrect at surgery or necropsy.

Two children had virtually no $\mathrm{CD} 3$ positive cells and both died. With the exception of these two cases none of the children had severe infections related to immunodeficiency, though frequent common infections occurred up to 2 or 3 years of age followed by spontaneous improvement.

Absolute numbers of $\mathrm{T}$ lymphocytes (CD3 antigen positive) were between 15 and $50 \%$ of normal in the majority of cases. While the absolute counts of both CD4 antigen positive and CD8 antigen positive were reduced, the low lymphocyte count was largely explained by the CD4 count. Formal phytohaemagglutinin studies were carried out in only a minority of children. However, a poor response was often noted to the phytohaemagglutinin stimulation carried out for karyotyping.

\section{Facial features}

While all of the children had some dysmorphic features these had not been noted by the referring clinician in a number of cases. Sometimes this was because a neonate required assisted ventilation and facial features were obscured, as shown in fig 1 . In other cases it was because of the subtlety of the dysmorphic features.

Lateral displacement of the inner canthi was a frequent finding. The palpebral fissures were often short and sometimes narrow. However, the angle of the palpebral fissures was not a useful feature as horizontal, upward, and downward slanting palpebral fissures were all seen (fig 2).

The root and bridge of the nose were wide and prominent. There was a marked indentation on either side of the nasal tip above the midpoint of each nostril. The philtrum was often short and poorly modelled but this was not a consistent finding, as can be seen from fig 3.

In the younger children the mouth was often small but this was not so noticeable in the older cases. The lips were thin in a third of cases.

The ears were low set and posteriorly rotated with deficient upper helices along with an increase in anteroposterior diameter giving a relatively circular ear (fig 4).

Most of the features were quite variable. The features that we found most helpful were the ear shape, prominent nasal root, and, in the 


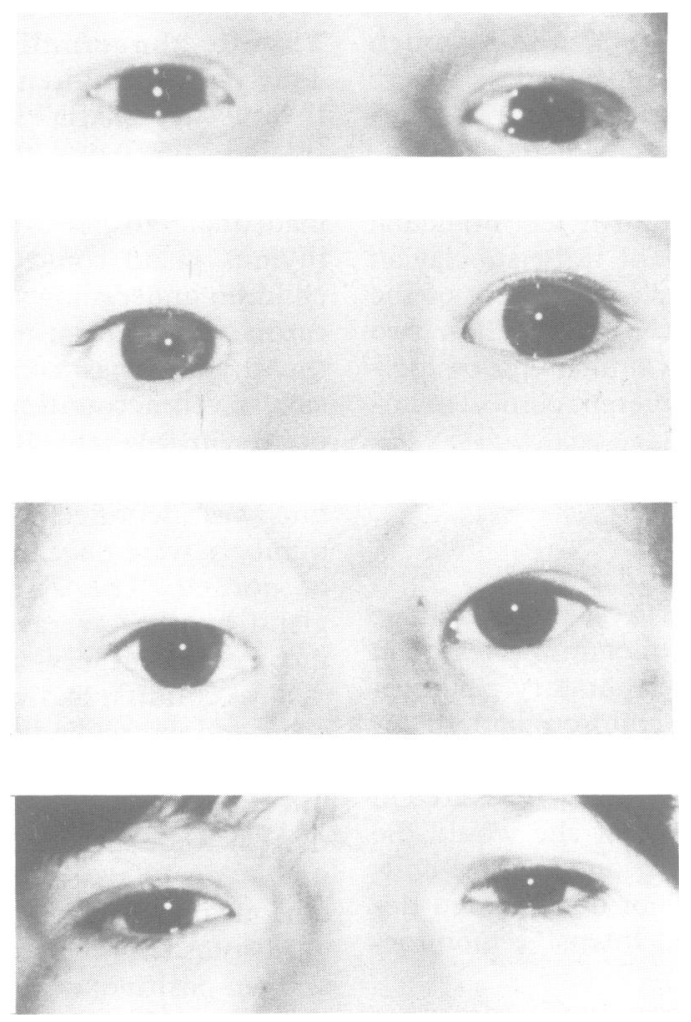

Figure 2 Nasal root and eyes in a baby (top) and infant with DiGeorge syndrome, a young child with a heart defect owing to monosomy 22q11, and a teenager with velocardiofacial syndrome (bottom).

younger child, the small mouth. The child shown in fig 5 is representative of the cases, she has most of the features discussed above, but is an attractive child who is not obviously dysmorphic.

\section{Other features}

Twenty-three children in this study had additional features (table 2). Many of the children in the study were too young for some of the complications to have become obvious. For example, nine children were old enough to present with symptoms of deafness; four of these did have symptoms and hearing deficit was confirmed on formal testing. Palatal or
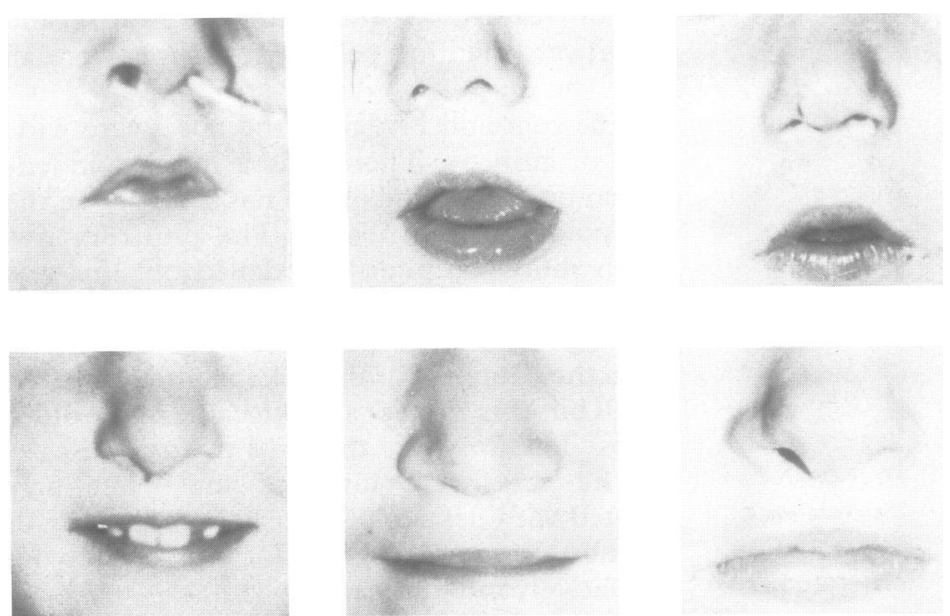

Figure 3 The tip of the nose, philtrum, and mouth. Age increases from neonate to young child on the top row and on into teenage years on the bottom row. Length of philtrum, size of mouth, and thickness of lips are variable.
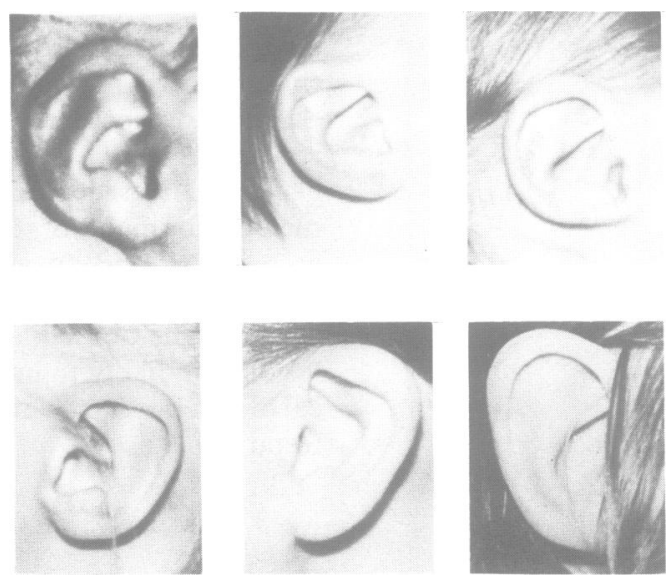

Figure 4 Abnormalities of the ear. The photographs show the appearance from neonate (top left) to adult (bottom right).

pharyngeal abnormalities were documented in 11 cases though this may again be an underestimate. Renal abnormalities were present in five cases. Two cases were hypothyroid. Three cases had unilateral facial nerve palsy. The age distribution, severity of heart malformation, and high mortality made a meaningful assessment of height potential and intellect difficult. An estimate of the developmental status was made on 13 surviving children between 2 and 16 years of age. Three children did not have any significant development delay and had an average academic ability. Two children attended normal school but required additional assistance. Two children had mild to moderate delay and six children had severe delay, three of whom had had significant hypoxic episodes in the perioperative period.

\section{Prognosis}

Twenty-four children were first assessed during the first few weeks of life. All had a cardiac

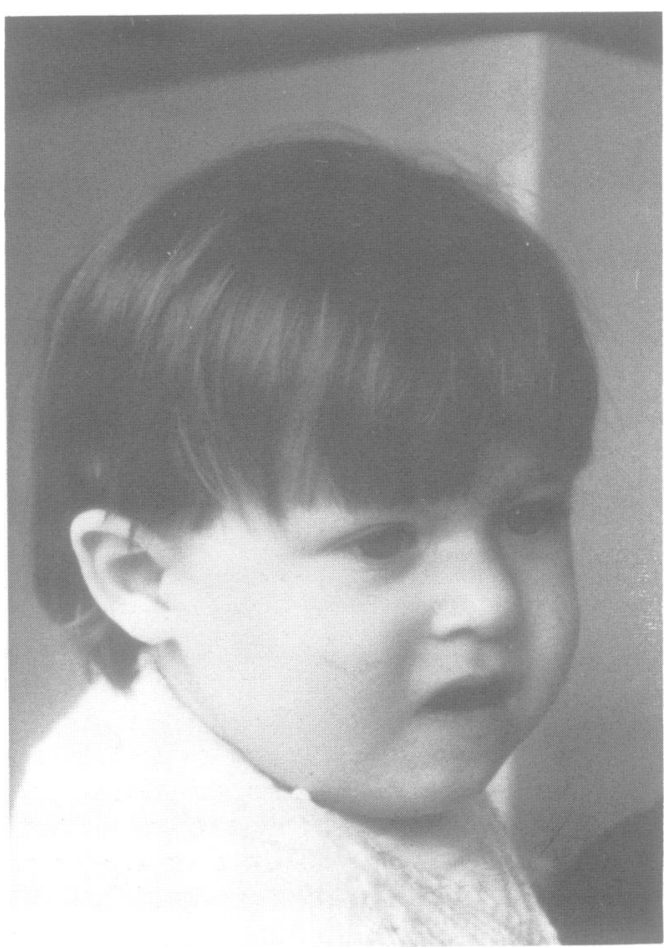

Figure 5 A child with DiGeorge syndrome. 
Table 2 Associated abnormalities.

\begin{tabular}{lll}
\hline Neural abnormalities & Bilateral & \\
Sensorineural deafness & Unilateral & 1 \\
Conductive deafness & & 1 \\
Cerebral hypoplasia & 1 \\
Microcephaly & 1 \\
Unilateral facial nerve palsy & 3 \\
Suspected hypopituitarism & 1 \\
Renal/urogenital & 3 \\
Unilateral renal agenesis & 2 \\
Unilateral dilated ureter & 1 \\
Dilated renal calyces & 1 \\
Undescended testes & \\
Musculoskeletal & 2 \\
Scoliosis & 2 \\
Talipes & 1 \\
Pectus excavatum & 1 \\
Joint laxity & 1 \\
Right lobster claw deformity & 2 \\
Palatal & \\
Bilateral cleft lip and hard palate & 3 \\
Cleft soft palate & 1 \\
Submucous cleft & 2 \\
High arched palate & 3 \\
VCFS speech without cleft & 2 \\
Miscellaneous & 1 \\
Hypothyroidism & 1 \\
Coloboma & 1 \\
Anteriorly placed anus & 1 \\
Umbilical hernia & 1 \\
Single umbilical artery & 1 \\
Bilateral single palmar creases & \\
Coronal synostosis & \\
\hline &
\end{tabular}

defect. Thirteen of these 24 children have died, 11 as a consequence of their cardiac defect and two secondary to immunodeficiency.

In the survivors where development was assessed, about half had moderate to severe delay. In half of those with severe developmental delay there had been significant hypoxic episodes at the time of operation that could have contributed to this.

\section{Recurrence risk}

Parental DNA was analysed in 15 of the deleted cases in this study. Deletions within chromosome $22 \mathrm{q} 11$ were identified in four mothers and no fathers. None of the four mothers had a cardiac defect or a history of convulsions or frequent infections in childhood. However, each of these mothers did have dysmorphic features associated with monosomy 22q11. One of them had two previous children with heart defects.?

We have not observed any sibs with a deletion in the absence of a parental deletion though the numbers of later born sibs remain small.

\section{Discussion}

This series of patients was selected on the basis of the DiGeorge phenotype. Criteria for inclusion were three of the following: congenital heart defect, hypoparathyroidism, T lymphocyte abnormalities, and dysmorphic facies.

The cardiac abnormalities were predominantly obstructive lesions of the outflow tract, particularly IAA. However, the spectrum of defects ranged from anatomical abnormalities of the subclavian artery with no clinical significance to left hypoplastic heart. Of note is the absence of abnormal venous connections in this group of children.

In four of the children the presenting feature was hypocalcaemic seizures. Almost a third of the total cases had a hypocalcaemic convulsion, underlining the importance of monitoring serum calcium in this group. While hypocalcaemia developed in the first two weeks of life and was transient in the majority, the fact that one child first became hypocalcaemic at the age of 5 years shows that the clinician must always be alert to this possibility. Our clinical impression is that the hypocalcaemia is transient but longitudinal studies are needed to clarify this. It would also be interesting to monitor calcium changes after acute illnesses in children in whom the calcium levels had returned to normal. Management of hypocalcaemia and hypoxia are obviously important to minimise later developmental problems.

Interpretation of thc $T$ lymphocyte data is not easy as normal ranges have not been established for these age groups and it is known that these parameters do change with age. However, 33 children had either low $T$ cell numbers or evidence of thymus hypoplasia. Two of the group had virtually no $T$ cells and both died. It is widespread practice to give irradiated blood products perioperatively to children who have cardiac lesions typical of DGS unless adequate $T$ cell numbers have been documented because of the small risk of graft versus host disease developing after transfusion of whole blood, plasma reduced blood, and platelet transfusions containing immunocompetent cells. The vaccination of children with DGS with live vaccines is an important consideration. These vaccines are potentially fatal in children with significant immunodeficiency. Equally important is the drawback of withholding a vaccine from children with congenital heart disease who should receive them. The relative risks and merits of vaccination should be considered in each case. As with the case of parathyroid function, longitudinal studies are required.

Hearing deficits were identified in five cases in this study of largely very young children, and so we would recommend a formal assessment of hearing. Five cases also had significant renal abnormalities and again we would recommend screening for renal anomalies and increased surveillance for urinary tract infections.

Counselling a couple who have a child with DGS depends on whether either parent has a deletion. So far there have been no documented recurrences in the absence of a parental deletion. We counsel a negligible recurrence risk to such couples. Our limited data suggest that in approximately $25 \%$ of couples a deletion is present. For these couples the risk of a further pregnancy with monosomy 22q11 is obviously $50 \%$. However, children with lethal DGS represent one end of a broad clinical spectrum. It may be argued that the possibility of there being few adverse consequences make it inappropriate to use deletion analysis as the sole basis for intervention in pregnancy. Conversely, even in the absence of the severe DiGeorge phenotype, significant clinical problems may ensue. Further data on the effects of maternal transmission, parental 
origin of deletions, and the possibility of genetic anticipation are needed to clarify these issues.

In his original report DiGeorge focused on thymic hypoplasia and hypocalcaemia. The clinical spectrum was widened to include heart defects and dysmorphic facial appearance. In 1976 Kinouchi and Takao reported a group of children with hypertelorism, narrow palpebral fissures, small mouth, nasal speech, and outflow defects of the heart in Japanese cardiology publications and suggested the term conotruncal anomaly face. ${ }^{8}$ They also suggested that $10 \%$ of Japanese children with tetralogy of Fallot have similar facial features. The Australian cardiologist Radford made the observation that children with truncus arteriosus and aortic arch defects but no thymic or parathyroid involvement also often have similar facial features. ${ }^{910}$ In 1978 Shprintzen et al reported a group of children with overt or submucous clefting of the palate, cardiac abnormalities, and developmental delay in cleft palate publications. ${ }^{112}$ Approximately $10 \%$ of this group develop psychiatric illness in adulthood. The dysmorphic features of all of these conditions show considerable overlap and deletions within chromosome 22q11 have now been identified in DGS, velocardiofacial syndrome, conotrucal anomaly face, and cases of isolated congenital heart disease ${ }^{3-5}$ 13-15 $^{15}$ (see also the paper by Burn et al on p 822). We think that these conditions are all part of one clinical spectrum and that the diagnostic label depends upon the age of presentation and the predominant clinical manifestation. We propose that each phenotype retains its established name but that an encompassing term for referring to the group as a whole should be CATCH $22 .{ }^{16}$

We thank all the families who have helped in this work and the clinicians who have referred them. The work has been supported by The British Heart Foundation, MRC, and The Borwick Trust. We also wish to thank Dr C Oley for her helpful comments on the dysmorphic features and Oonagh Claber for her help in preparing the manuscript.

1 Cooper MD, Peterson RDA, Good RA. A new concept of the cellular basis of immunity. F Pediatr 1965;67:907-8.

2 De la Chapelle A, Herva R, Koivisto M, Aula P. A deletion in chromosome 22 can cause DiGeorge syndrome. Hum Genet 1981;57:253-6.

3 Scambler PJ, Carey AH, Wyse RKH, et al. Microdeletions within 22q11 associated with sporadic and familial DiGeorge syndrome. Genomics 1991;10:201-6.

4 Driscoll DA, Budarf ML, Emanuel BS. A genetic etiology for DiGeorge syndrome: consistent deletions and microdeletions of 22q11. Am f Hum Genet 1992;50:924-33.

5 Carey AH, Kelly D, Halford S, et al. Molecular genetic study of the frequency of monosomy 22q11 in DiGeorge syndrome. Am f Hum Genet 1992;51:964-70.

6 Wilson DI, Bennett Britton S, McKeown C, et al. Noonan's and DiGeorge syndromes with monosomy 22q11. Arch Dis Child 1993;68:187-9.

7 Wilson DI, Cross IE, Goodship JA, et al. DiGeorge syndrome with isolated aortic coarctation and isolated ventricular septal defect in three sibs with a 22q11 deletion of maternal origin. Br Heart f 1991;66:308-12.

8 Takao A, Ando M, Cho K, Kinouchi A, Murakami Y. Etiological categorization of common congenital heart disease. In: Van Praagh R, Takao A, eds. Etiology and morphogenesis of congenital heart disease. New York: Futura, 1980:253-69.

9 Radford DJ. Spectrum of DiGeorge syndrome in patients with truncus arteriosis: expanded DiGeorge syndrome. with truncus arteriosis: expanded
Pediatr Cardiol 1988;9:95-101.

10 Radford DJ. Truncus arteriosis and facial dysmorphism. Aust Paediatr f 1985;21:131-3.

11 Shprintzen RJ, Goldberg RB, Lewin ML, et al. A new syndrome involving cleft palate, cardiac anomalies, typical facies, and learning disabilities: velo-cardio-facial syndrome. Cleft Palate f 1978;15:56-62.

12 Shprintzen RJ. Goldberg RB, Young D, Wolford L. The velo-cardio-facial syndrome: a clinical and genetic analysis. Pediatrics 1981;67:167-71.

13 Scambler PJ, Kelly D, Lindsay E, et al. Velo-cardio-facial syndrome associated with chromosome 22 deletions encompassing the DiGeorge locus. Lancet 1992;339:1138-9.

14 Wilson DI, Goodship JA, Burn J, Cross IE, Scambler PJ. Deletions within chromosome 22q11 in familial congenital heart disease. Lancet 1992;340:573-5.

15 Driscoll DA, Spinner NB, Budarf ML, et al. Deletions and microdeletions of 22q11.2 in velo-cardio-facial syndrome. Am 7 Med Genet 1992;44:261-8.

16 Heller J. Catch 22. London: Jonathan Cape, 1962. 